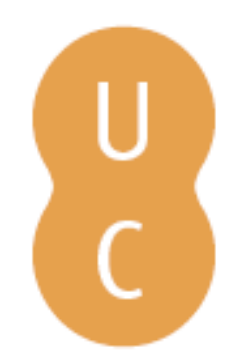

\title{
nommalina
}

\section{Le fantastique comme catégorie esthétique: contaminations entre fantastique et grotesque}

\author{
Autor(es): $\quad$ Simões, Maria João \\ Publicado por: Imprensa da Universidade de Coimbra \\ URL \\ persistente: URI:http://hdl.handle.net/10316.2/38700 \\ DOI: $\quad$ DOI:http://dx.doi.org/10.14195/978-989-26-1164-8_23 \\ Accessed : $\quad$ 26-Apr-2023 15:18:25
}

A navegação consulta e descarregamento dos títulos inseridos nas Bibliotecas Digitais UC Digitalis, UC Pombalina e UC Impactum, pressupõem a aceitação plena e sem reservas dos Termos e Condições de Uso destas Bibliotecas Digitais, disponíveis em https://digitalis.uc.pt/pt-pt/termos.

Conforme exposto nos referidos Termos e Condições de Uso, o descarregamento de títulos de acesso restrito requer uma licença válida de autorização devendo o utilizador aceder ao(s) documento(s) a partir de um endereço de IP da instituição detentora da supramencionada licença.

Ao utilizador é apenas permitido o descarregamento para uso pessoal, pelo que o emprego do(s) título(s) descarregado(s) para outro fim, designadamente comercial, carece de autorização do respetivo autor ou editor da obra.

Na medida em que todas as obras da UC Digitalis se encontram protegidas pelo Código do Direito de Autor e Direitos Conexos e demais legislação aplicável, toda a cópia, parcial ou total, deste documento, nos casos em que é legalmente admitida, deverá conter ou fazer-se acompanhar por este aviso.

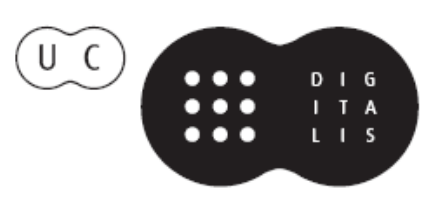


Maria João Simões

Universidade de Coimbra / Centro de Literatura Portuguesa

\section{LE FANTASTIQUE COMME CATÉGORIE ESTHÉTIQUE: CONTAMINATIONS ENTRE FANTASTIQUE ET GROTESQUE}

L'attrait du fantastique dans l'art actuel — visible dans plusieurs domaines artistiques, soit-il dans le cinéma, soit dans la bande déssinée, soit encore dans la littérature digitale ${ }^{1}$ - a obligé la critique à rompre les frontières avec lesquelles elle a voulu cerner l'empreinte fantastique. On reconnaît, aujourd'hui, non seulement la difficulté de définir le territoire du fantastique et ses frontières vis-à-vis possibles débordements de sens vers des termes voisins, mais aussi la difficulté de réussir à une équivalence entre ces désignations voisines et leur champ de référence en ce qui concerne des langues différentes - notamment les équivalences ou différences entre les désignations en langue anglaise et allemande et les désignations des langues néo-latines.

1. Les problèmes de terminologie. La diversité terminologique engendre des problèmes de traduction qui se répercutent inévitablement dans la critique littéraire. Une excellente mise au point sur ce problème est achevée par Arnaud Huftier et Roger Bozzetto dans un des premiers chapitres de l'œuvre Frontières du Fantastique, où l'histoire de la critique littéraire française sur le fantastique et sa répercussion dans d'autres pays est scrutée en détail permettant de voir les détours et les adaptations (souvent trop forcés) utilisés au service d'un cadre théorique choisi préalablement. Un cas paradigmatique de ce genre de problèmes

\footnotetext{
1 Par exemple, une des premières histoires de littérature digitale et une des plus citées, Patchwork Girl, reformule le mythe fantastique de l'homme fabriqué proposant une version féministe du Dr. Frankenstein (cf. http://www.eastgate.com/catalog/PatchworkGirl.html).
} 
est la fortune de la théorisation de Tzvetan Todorov qui a répandu les appropriations et les adaptions réductrices faites par l'auteur, lesquelles, selon Arnaud Huftier, camouflent des différences et des manques de correspondance entre les langues des auteurs convoqués par T. Todorov. La répercussion inusitée de l'œuvre todorovienne (visible par le grand nombre de traductions) a propagé la mainmise de son auteur en ce qui concerne les mots-clefs du fantastique en allemand et en anglais.

2. Les différentes Histoires. Ce premier problème révèle alors un autre celui qui concerne l'histoire du fantastique et la question de ses origines: peut-il y a avoir une histoire du fantastique ou aura-t-il plutôt plusieurs histoires du fantastiques dépendantes des perspectives critiques ou théoriques qu’on décide adopter? Il est indéniable aujourd'hui que la perspective diachronique a été aussi souvent manipulée de façon à justifier certaines positions ou divisions théoriques.

La perspective théorique qui considère le fantastique comme un "genre" (et, en plus, un genre récent) est assez répandue et peut-être trop bien ancrée dans l'univers scolaire et académique, surtout après la riche fortune que l'œuvre de T. Todorov y a eu. Selon cette perspective le fantastique serait le contrepoint de l'esprit scientifique héritier de la révolution copernicienne qui se rend visible à outrance pendant le positivisme - une idée défendue par Roger Caillois et maintes fois reprise et répétée comme nous dit Francis Dubost (1991: 120) qui, d'une certaine manière, la conteste.

Cependant, si l'on envisage une perspective diachronique vraiment élargie — et qui ait en ligne de compte l'ouverture possibilitée par la littérature comparée - il faut penser à des productions si diverses que celles influencées par la tradition grecque, perse et chinoise, la tradition médiévale, celtique ou nordique, qui, surtout à travers une contamination avec la fantasy ${ }^{2}$ et avec le merveilleux, iront influencer le fantastique contemporain ${ }^{3}$. Il est éclaircissant, à ce propos, la

\footnotetext{
2 Un écho contemporain de ce transfert historique peut être perçu dans la fantaisie de l'œuvre The Lord of the Rings de J. Tolkien, qui a été inspiré par des légendes médiévales et par le poème hérö̈que Beowulf de thématique nordique.

3 J. Ziegler (1985) affirme que les écrivains de fantaisies ont trois alternatives: "First they can trivialize or make banal the reality around them. This is the solution of most contemporary fantasists. Second they can escape from the horrors of the present by creating their own world (...). Third, they can illuminate the present by exagerating its flaws and absurdities. (...)".
} 
réflexion faite para Borges, dans le texte "Kafka y sus precursores", où il fait "un examen des précurseurs de Kafka”, confessant les avoir retrouvés d'une façon curieuse — c'est à dire, à rebours:

A éste [Kafka], al princípio, lo pensé tan singular como el fénix de las alabanzas retóricas; a poco de frecuentarlo, creí reconocer su voz, o sus hábitos, en textos de diversas literaturas y diversas épocas. (Kafka, 1985: 115).

Outre la ressemblance qu'il découvre entre le paradoxe de Zenon et le motif du roman Le Château, il écoute aussi des échos de Kafka dans un apologue du proseur chinois Han Yu Si et voit une "prophétie" de l'œuvre de Kafka dans le poème Fears and Scruples de Robert Browning, de 1876. Cette réflexion - qui est simultanément un aveu de ses propres influences - a le mérite d'éclaircir comment le (souvent dit) nouveau fantastique est fait de plusieurs mélanges et de diverses influences à l'image de ce qui se passe avec le "réalisme magique". Borges nous montre que l'Histoire a ses tours et ses détours - elle est complexe.

En effet, comme nous dit Francis Dubost, beaucoup de productions artistiques posent des problèmes à l'établissement d'une date tranchante pour le début du fantastique (soit-il 1764 avec le Château d'Otranto de Horace Walpole, soit 1772 avec le Diable Amoureux de Jacques Cazotte). Francis Dubost énumère, à ce propos, une série d'œuvres littéraires qui questionnent ces dates. Mais il faut élargir ce questionnement à des exemples d'autres domaines artistiques, une fois que le fantastique n'existe pas seulement dans la littérature. Que dire alors de certaines illustrations du Commandeur de Don Juan (faites à partir de 1630), ou de certaines illustrations du "Dr. Faust", et de la représentation de l'au-delà dans l'eau-forte "Un alchimiste au travail" (souvent appelé "Dr. Faust") de Rembrandt, datées de 1652? Comment peut-on classer certains tableaux manifestement fantastiques comme le "Paysage Nocturne Fantastique" de Marco Ricci, daté de 1708? En vérité le fantastique ne se laisse pas coincer à une époque, pourvu qu'il ne soit pas envisagé au-dedans des murs du "genre". Certes il y eut, au dix-neuvième siècle, une autonomisation progressive de cette catégorie esthétique cheminant, par opacification, vers le genre (le fantastique gothique), mais ceci ne veut pas dire qu'il n'y aurait eu déjà des effets de fantastique auparavant 
et ne veut pas dire non plus qu'il ne puisse pas exister de fantastique dans des textes qui ne peuvent pas être considérés comme impartis au fantastique en tant que genre ${ }^{4}$.

3. Taxonomie et caractérisation. Si, par contre, on envisage le fantastique essayant une perspective synchronique on peut constater la diversité de théories et de manières de concevoir le fantastique. Outre ceux qui persistent à la notion de fantastique définit comme genre, il y a plusieurs approches du fantastique: Roger Bozzetto parle de "sentiment" et d' "aspects", Lucie Armitt et Remo Ceserani de "mode", A. Huftier de "modulations" (Bozzetto et Huftier, 2004: 43). La plupart de ces critiques envisagent leurs désignations les situant à un niveau différent de celui du genre, par rapport à la taxonomie littéraire la plus répandue qui présuppose la différence entre modes et genres.

Il faut, alors, comprendre le statut de cet autre niveau. Pour y parvenir il semble indispensable de voir comment le fantastique est abordé dans d'autres domaines artistiques et à quelles autres catégories il est rapproché dans l'analyse de l'Esthétique en tant que discipline.

Dans le Vocabulaire d'Esthétique d'Étienne Souriau, on rencontre le concept de catégorie esthétique au sein duquel sont groupées les notions de grotesque, sublime, burlesque, fantastique, etc. Les éléments constitutifs qu'on y attribue au concept de catégorie esthétique sont trois: un ethos particulier, un système de forces organisées et une valeur esthétique spéciale. À son tour cet ethos implique "une atmosphère affective spécialisée", le système implique "l'agencement des éléments dans une relation et interaction organique" et la valeur indique "une variété particulière d'idéal esthétique" (Souriau, 1990). Il s'agit, comme on dirait aujourd'hui, d'une catégorie multidimensionnelle et interrelationnelle qui rend compte de la complexité du phénomène. Un autre esthéticien, Robert Blanché, a aussi étudié ce concept dans une œuvre précisément intitulée Catégories Esthétiques, de 1979, où il complète le diagramme proposé par É. Souriau, disposant les catégories sur une rosace dont les lignes radiales indiquent des proximités et des oppositions:

\footnotetext{
4 Comme exemple on peut penser au conte "Le manteau" de Gogol, un conte qui, pendant trois quarts de l'intrigue, obéit aux stratégies réalistes, mais qui présente des éléments fantastiques à la fin de la narrative.
} 


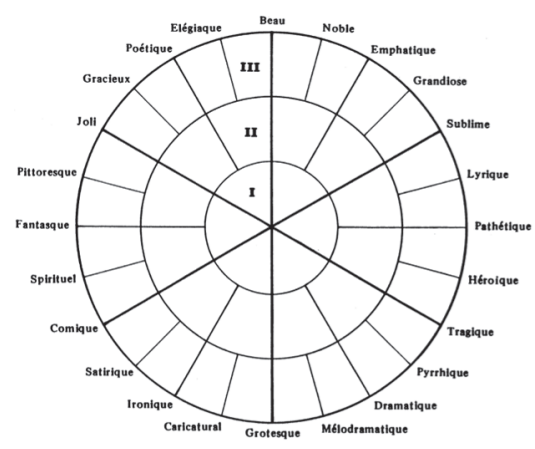

Robert Blanché nous avertit sur l'impossibilité d'épuiser une liste définitive des catégories et souligne encore la perméabilité entre les différentes catégories. Ce terme 'catégorie' est, cependant, dangereux à cause de la polysémie et du poids philosophique qu'il présente. Mais, on peut rencontrer des désignations conceptuelles qui recouvrent (partielle ou approximativement) cette notion, si on pense, par exemple, aux "essentialités" ou "qualités dérivées" de Roman Ingarden ou aux "modes dérivés" de Mikhaïl Bakhtine. On retrouve aussi ce problème conceptuel dans les discussions d'esthéticiens comme Frank Sibley et Gérard Genette quand ils parlent de propriétés ou de prédicats des œuvres d'art. Gérard Genette (faisant allusion à l'origine kantienne ${ }^{5}$ de la désignation 'prédicats') met en relief les composants objectifs et subjectifs des prédicats, suggérant la capacité d'objectivation des prédicats capables d'augmenter leur propre opacité ${ }^{6}$. Or, ce mouvement d'objectivation peut aider à expliquer le glissement du caractère qualitatif de ces catégories à la caractérisation constitutive du genre, c'est à dire le passage du tragique à la tragédie, du comique à la comédie, du fantastique-qualité (ou prédicat) au fantastique-genre.

5 Selon Kant, "Aesthetic, just like theoretical (logical) judgements, are divisible into empirical and pure. (...) the second those by which beauty is predicated of an object or its mode of representation. The former are judgements of sense (material aesthetic judgements), the latter (as formal) alone judgements of taste proper. (...) To form a cognitive judgement we may immediately connect with the perception of an object the concept of an object in general, the empirical predicates of which are contained in that perception. In this way, a judgement of experience is produced. (...)

But we may also immediately connect with a perception a feeling of pleasure (or displeasure) and a delight, attending the representation of the object and serving it instead of a predicate." (http://etext.lib.virginia.edu/toc/modeng/public/KanJudg.html).

6 Selon G. Genette (1997: 114), "les qualifications "esthétiques", qui font en quelque sorte passer leur caractère appréciatif sous un couvert descriptif, constituent de très efficaces opérateurs d'objectivation". 
La considération de ces caractéristiques de profil qualitatif ${ }^{7}$ ou adjectival $^{8}$ est essentiel pour l'appréciation esthétique des œuvres d'art, soit-elle celle du sens commun soit du critique érudite. On voit facilement que les catégories littéraires traditionnelles sont plutôt descriptives (et souvent trop circonscrites) et, quoique leur visée soit la description, son utilisation tend à (ou tombe presque dans) l'évaluation. Mais, la réalité des propriétés attribuées aux œuvres artistiques est beaucoup plus complexe - comme on peut voir par l'analyse des propriétés esthétiques évaluatives ("evaluative aesthetic properties") faite par Alan D. Goldman (1995: 17) dans l'œuvre Aesthetic Value. Cet esthéticien fait une énumération des diverses sortes de termes utilisés par les récepteurs et la critique dans l'évaluation: termes évaluatifs, formels, évocatoires, comportementaux, représentationnels, termes perceptuels de second ordre et termes historiques. Que l'on accentue l'un ou l'autre, cela dépend du choix: dans un acte évaluatif on peut mettre l'accent sur les comportements, sur les caractéristiques formelles, ou sur les caractéristiques représentationnelles. Considérant ces distinctions, on peut rencontrer une explication pour le manque de consensus et la polémique au sujet de savoir quel "effet de fantastique" est la condition sine qua non de sa présence, qui, comme on le sait, peut aller de l'horreur à l'effrayant, de l'incertitude à l'épouvante, de la peur à l'incroyable, du sursaut au dégoût, etc.. Dès qu'on pense à des exemples concrets, on a du mal à choisir un seul effet de fantastique pour qu'il y ait du fantastique - il suffit de penser à La Métamorphose de Kafka pour que l'on rencontre, tout de suite, plusieurs effets. Comme Alan H. Goldman (1995: 18) nous avertit, si certains de ces types de propriétés sont phénoménologiquement perceptibles, d'autres dépendent de la "connaissance des contextes et des facteurs causaux externes à l'œuvre". L'incontournable analyse de A. Goldman, si minutieuse et si juste, chemine vers la difficile question de la portion de subjectivité et/ou objectivité comprise dans l'évaluation des ouvres (dépendante des propriétés). En ce qui concerne les objectifs et les préoccupations proposés pour ce texte-ci, le plus important est de retenir l'idée de pouvoir abstraire des propriétés à partir des relations que les œuvres

\footnotetext{
7 La "qualité" - une des catégories analysées par Aristote — était une des catégories enseignées, en latin, avec la désignation de "praedicamentum (-a)", ce qui met en évidence sa racine de prédicat proche de la signification de "praedicare".

8 Rappelons ce que A. Fowler disait dans son ouvre Kinds of Literature. An Introduction to the Theory of Genres and Modes: "modal terms tend to be adjectival" (Fowler, 1982).
} 
entretiennent entre elles et avec nous, et aussi à partir des relations que les œuvres ont avec le contexte qui les entoure.

4. Catégories et complexité. En fait, outre les relations de proximité et contamination ${ }^{9}$ que le fantastique subit et qu'il engendre relativement aux catégories voisines, en ce qui concerne la question de l'appréciation et de l'évaluation esthétique du fantastique il est très important de les penser avec l'aide du paradigme de la complexité ${ }^{10}$ et des raisonnements de la pensée complexe, une fois que le fantastique se constitue en tant que propriété appartenant à différents niveaux de conceptualisation et à plusieurs espèces catégorielles ${ }^{11}$. Dans ce sens, il faut donc penser comme le fantastique émerge d'une création vouée à la complexité complexité dont les principes fondamentaux, selon Edgar Morin, sont le principe hologrammatique, le principe dialogique et le principe de récursion. Cela veut dire que toutes les stratégies du fantastique et tous les éléments du fantastique qu'on peut énumérer, grouper, relier et analyser — sachant qu'il y aura toujours la possibilité d'ajouter un nouvel élément — tous ces éléments et stratégies, on disait, doivent, eux aussi, être considérés dans la multiplicité des ces relations, c'est à dire dans sa complexité ${ }^{12}$.

5. Fictionnalité spécifique. Penser le fantastique en tant que concept complexe permet de voir qu'il inscrit comme un concept dépendant de plusieurs catégories lesquelles appartiennent à des dimensions différentes et qui, n'étant pas concentriques, s'interceptent, se croisent et se renvoient hologrammatiquement

9 Le fantastique émerge dans l'œuvre artistique comme une composante fluide, se rapprochant ainsi de la façon dont Lucie Armitt (1996: 3) envisage la fantasy, c'est à dire, en tant que mode "fluid, constantly overspiling the very forms it adopts, always looking, not so much to escapism but certainly to escape the constraints that critics like always and inevitably impose upon it.

10 Edgar Morin (1991: 291) nous dit: "Si nous pouvions imaginer un paradigme de la complexité, ce serait un paradigme qui se fonderait sur l'union de la distinction, qui est nécessaire pour concevoir des objets ou phénomènes, et la conjonction, qui est nécessaire pour établir les interrelations et les articulations. (...) Je dirai surtout que ce serait un principe dialogique."

11 On peut penser à la catégorie ou prédicat esthétique, ou au genre — soutenu par une opacité d'éléments organisés de façon plus figée comme dans le fantastique gothique - , ou à son type de protocole fictionnel, ou à l'espèce sémantique, etc..

12 Pour un diagramme représentationnel de la complexité des éléments et stratégies du fantastique voir Simões, 2006: 31. 
et réciproquement. Le fantastique se constitue, ainsi, comme un cas d'appartenance multidimensionnelle ${ }^{13}$. En ce qui concerne, par exemple, la fictionnalité, le fantastique suscite et engendre une simulation différente de celle des ouvres réalistes, il prescrit une croyance et un faire-croire particuliers, c'est à dire un spécial make-believe - cette dynamique expression utilisée par Kendall Walton dans sa théorie de la fictionnalité — aujourd'hui incontournable - exposée dans l'œuvre Mimesis as Make-Believe. Le fantastique exige ce que Kendall Walton appelle le "principe de la croyance mutuelle" (Mutual Belief Principle), une fois qu'il échappe aux constrictions mimétiques du "principe de réalité" ("Reality Principle") celui-ci essentiel à toute fiction ${ }^{14}$. À cette lumière on comprend facilement ce que dit Adolfo Bioy Casares dans Plan d'Évasion: "toute vision imaginaire devient réelle pour celui qui y croit".

Il s'agit, en effet, d'un protocole spécial celui qui doit suivre le lecteur de La Métamorphose quand il joue à croire que Gregor se transforme en vermine ou insecte (usuellement crû être un cancrelat ou une blatte). Ce procédé se repète dans une autre narrative de Kafka intitulée "Joséphine la cantatrice ou le peuple des souris" où le narrateur est un rat qui parle initialement d'une célèbre chanteuse, gloire de la nation, qui, au bout du compte — on le sait ensuite - ne chante pas, mais siffle, tout simplement, et, vers la fin de la narrative, on est informé qu'elle ne siffle plus: elle ne se digne même pas d'apparaître dans les concerts planifiés. Tout au long du conte le lecteur est obligé de refaire constamment l'ensemble de croyances qu'il est en train de construire, lesquelles se diluent progressivement laissant le lecteur pris à la déception. Le narrateur est un rat: cependant, moitié de son discours est anthropomorphique et nous fait oublier que, dans cette histoire, il s'agit de rats puisque ce narrateur-rat utilise le vocabulaire des humains, parlant de son "peuple" quand il se réfère aux rats et de Joséphine comme "une délicate personne"; l'autre moitié du discours, par le biais d'expressions ambiguës ou polysémiques, nous ramène à l'insolite de la situation d'un rat qui parle.

13 Certainement cette idée permet de dépasser les réticences colloquées par Roger Bozzetto à impartir "la fantastique" aux "catégories de la sensibilité" — dans le texte qui ouvre le numéro 611 de la revue Europe (Mars, (1980) 5) sur "Les Fantastiques". En effet si l'on conçoit le fantastique subissant une appartenance multidimensionnelle, on peut l'impartir à une catégorie de sensibilité et à un principe fictionnel spécifique (ou, encore, à d'autres catégories) simultanément.

14 D'une certaine façon, il s'agit d'une catégorisation sémantique fictionnelle qui recouvre les théories dites sémantiques du fantastique (voir Molino, 1980, 16-18). 
Le conte peut être lu comme une allégorie de l'éphémérité de la gloire artistique ou politique, laquelle se dissipe et s'épuise rapidement absorbée par l'oubli. L'atmosphère évanescente de la gloire est engrossée par l'absurde de la relation entre le peuple (de la nation des rats) et son idole, et, par là, le lecteur est conduit à faire un démontage de la supposée représentativité de l'idole-performeur relativement à la nation. Par le biais de ce démontage on atteint toute la force subversive qui s'insinue dans le texte en perforant la signification initiale.

6. Subversion et transgression. Presque unanimement on reconnaît, avec Roger Caillois, que "tout fantastique est rupture de l'ordre reconnu, irruption de l'inadmissible au sein de l'inaltérable légalité quotidienne".

Dans l'histoire de Joséphine, la capacité subversive du texte n'advient pas seulement de la stratégie d'utilisation de l'élément fabuleux de l'animal qui parle, mais aussi de ce constant minage du monde fictionnel qui vient juste d'être tracé. On peut trouver un exemple semblable dans un autre texte de Kafka intitulé "le Pont" où on peut lire la très courte narrative d'un pont qui parle et semble humain, parce qu'il est représenté (aussi) de façon anthropomorphique, avec mains, orteils et chevelure. Son sort semble être décidé par l'effroi causé par un homme qui marche en dessus: en s'inclinant pour guetter le mouvement de l'homme, le pont ne peut que tomber:

J'étais raide et froid. (...) soudain j'entendis un pas d'homme! Par ici, par ici! Allonge-toi, pont, apprête-toi (soutient le pas qui t'est confié. (...) Mais ensuite (...) il sauta les pieds joints sur le milieu de mon corps. Je fus saisi de frayeur (...) Qui était-ce? (...) Un enfant? Un suicidé? (...) Et je me renversais pour le voir. — Pont qui se renverse! Je n'étais pas encore tourné que déjà je tombais, (...) et déjà j’étais déchiré (...) (Kafka, 1945: 260).

Il s'agit ici d'une subversion de ce qu'on croit normalement et rationnellement - une subversion qui devient plus forte, on le sait, dans La Métamorphose, où le lecteur accompagne la transformation du personnage engendrant une situation qui, d'une certaine manière, le rend plus complice du jeu fictionnel. 
Un lecteur complice est demandé aussi pour suivre la stratégie fictionnelle utilisée par J. L Borges dans la narrative fantastique ${ }^{15}$ "Tlön, Uqbar, Orbis Tertius", une fois que le lecteur doit croire non seulement à l'existence d'une conversation entre Borges et Bioy Casares à propos de l'existence d'une entrée insolite dans un "livre imaginaire", de l'Anglo-American Cyclopedia ${ }^{16}$, mais il doit aussi croire (quoique à un autre niveau sémantico-narratif) à l'existence d'un endroit spécial — "Uqbar" - et il doit croire encore au monde imaginaire de "Tlön" (référés dans la dite Cyclopedia). En plus, dans ce texte aussi, le lecteur doit accompagner un subtil mouvement de démontage de toute cette dérision pour apprécier la dissolution de frontières fictionnelles y présentée qui s'origine par un renversement ambigu où le monde dessiné comme imaginaire absorbe le monde représenté comme normal ${ }^{17}$. Qu'il y a, dans ce sens, une prescription (pour utiliser la terminologie waltonienne) dirigée au lecteur pour suivre ce cheminement - et qu'elle est vraiment intentionnelle - cela devient très claire si on ose faire une généralisation à partir d'une symptomatique situation de création fictionnelle racontée par Borges au début de ce même texte. En effet, Borges relève un peu le voile de sa propre stratégie fictionnelle quand il fait allusion à une présomptive discussion entretenue avec Bioy Casares à propos de l'élaboration d'un "roman à la première personne, dont le narrateur puisse omettre et défigurer les événements et puisse tomber dans des contradictions diverses, qu'eussent permis à peu de lecteurs - à très peu de lecteurs — de deviner une réalité atroce ou banale" (Borges, 1998: 447).

Cette espèce d'estompage prémédité, ainsi que ce questionnement du principe fictionnel lui-même (qui appellerait un vertige "intellectuel") sont des caractéristiques relevées par certains critiques pour distinguer ce qu'ils appellent "le nouveau fantastique" (voir Bozzetto et Huftier, 2004: 37). Contestant la pertinence de cette étiquette et démontrant comme elle tend parfois à gommer "le real-maravilloso" (41), Arnaud Huftier nous incite à considérer ces caractéristiques comme des

15 L'adjectif fantastique est utilisé par Borges dans le Prologue de Fictions pour désigner toutes les narratives de ce volume à l'exception de la septième qu'il dit être policière. (Borges, 1998: 445).

16 L'expression "livre imaginaire" est utilisée par Borges (dans le Prologue de Fictions) pour désigner ce livre - un supposé volume de l'Anglo-American Cyclopedia dit être est une "reimpréssion littérale et divergente de l'Encyclopaedia Britannica de 1902". (Borges, 1998: 447).

17 C'est un cheminement et une stratégie différents de ceux que Borges utilise dans "Les ruines circulaires", où, selon Borges, "tout est irréel". (Borges, 1998: 445). 
"effets de fantastique" (idem, 45) choisis par les auteurs qui, d'une certaine façon, accompagnent une certaine évolution de la narrative contemporaine vers l'autoréflexivité.

Mais ce qui est plus intéressant c'est voir comment cet estompage et ces jeux avec les principes de la fictionnalité engendrent et/ou accentuent da "dualité" et la "duplicité" qui caractérisent, selon A. Huftier (idem, 47), les "effets de fantastique". Cette "bifacialité" est bien évidente dans la phrase de Borges citée quand il choisi joindre les deux verbes "omettre et défigurer" pour rendre compte des "événements" d'une narration, ainsi que les deux adjectifs "atroce" ou "banale" pour rendre compte de la "réalité" représentée. La contradiction de ces deux pairs de mots nous annonce la transgression de frontières engendrée par le fantastique dans sa gestion de la duplicité.

7. Contamination avec le grotesque. En aval de cette duplicité qui trouble et subvertit la catégorisation ${ }^{18}$ linéaire, nous devons nous attarder aussi sur le choix des mots "défiguré" et "atroce". Ces mots révèlent une tendance du fantastique vers l'horrible, insinuant la face déformée ou laide de l'inconnu l'inquiétante différence de l'irréférent proposé au public (voir Chareyre-Méjan, 1998: 106) - formant une espèce de boucle: si l'inconnu est horriblement inquiétant, alors l'horrible et le déformé peuvent être un bon choix pour représenter l'inconnu. Mais s'il cherche le laid, comment expliquer, alors, l'attrait du fantastique?

Là aussi, une ancienne discussion réapparaît, continuant, de nos jours, à appeler l'attention de esthéticiens: la question de l'appréciation esthétique et sa liaison avec le plaisir esthétique - une question indissolublement liée à une autre: celle de l'identification des propriétés de l'œuvre d'art et son rôle dans

18 En étudiant la perception visuelle, Daniel Chandler met en évidence l'importance de la catégorisation: "Categorization is a key 'top-down' process which is involved in perception. Like schemata, categories simplify. Categorization has a number of functions: it makes complexity manageable; it speeds up recognition; it reduces effort and learning; it makes the most of past experience; it enables the inferences about further attributes (going beyond what is 'given'); it makes events predictable; it supports systematization; it bonds social behaviour (providing shared frameworks); it tailors the world to our purposes; it makes the world seem more meaningful. The cost of these advantages is a loss of particularity and uniqueness in perception and recall." (cf. http://www.aber. ac.uk/media/Modules/MAinTV/visper05.html). 
l'appréciation et le plaisir esthétiques ${ }^{19}$. Récemment, James C. Anderson (70), a contesté l'idée d'une liaison directe entre les concepts de appréciation esthétique, plaisir esthétique et valeur esthétique. Or, cette mise en question est très importante parce que, d'une certaine façon, le fantastique et le grotesque mettent en question la relation directe entre les deux concepts: appréciation esthétique-plaisir esthétique. En fait, la quête de l'insolite et de l'anormal les éloignent du beau (trop souvent compris comme l'essence de l'esthétique), du gracieux et même de l'amusant - sources claires du plaisir. Mais, comme nous dit James C. Anderson (2000: 71), "l'appréciation esthétique n’implique nécessairement pas le plaisir", mais découle plutôt de "croire l'expérienciation (le sentir) des propriétés d'un objet comme étant intrinsèquement valable"20.

En fait, le gros de la valeur esthétique attribuée au fantastique lui advient de sa capacité de jouer avec les catégories connues et du brouillage catégoriel qu'il apporte. Or, il y a un autre prédicat, le grotesque, qui opère sur le même procédé.

Le grotesque, selon Dieter Meindl, émerge de la "combinaison tensionnelle d'éléments attrayants et répulsifs" ${ }^{21}$, il se situe à l'intersection de plusieurs sphères, et, étant axé sur l'ambivalence ${ }^{22}$ et sur la contradiction ${ }^{23}$, il questionne la signification simple et linéaire. De plus, comme le souligne Frederick Burwick (1990: 128-9), une des plus importantes fonctions du grotesque est de nous donner "l'illusion du tromper" ou de la simulation ("the illusion of delusion"), attirant

19 Il faut penser, en ce qui concerne l'appréciation et le plaisir esthétiques, aux caractéristiques des œuvres d'art et leurs différences vis-à-vis celles de la beauté naturelle. Récemment, philosophes de l'art comme Noël Carroll et Kendhall Walton sont revenus à ces questions et ont proposé des conceptualisations qui ont été revisitées par James C. Anderson. Cet auteur soutient quelques unes des positions des esthéticiens mentionnés, mais met en évidence la distinction nécessaire vis-à-vis le concept d'évaluation et de plaisir esthétique.

20 Selon J. C. Anderson (2000, 71), "what is necessary for aesthetic appreciation is the recognition or judgment 'that something is good'. (...) if we understand aesthetic appreciation as a species of value judgment which focuses on one's experience of objets and their properties. We can, thus define aesthetic appreciation as believing the experience of the properties of an object to be intrinsically valuable."

21 Dieter Meindl (1996: 14) met en évidence le consensus de la critique relativement a cet aspect: "... notwithstanding the multiplicity of scholarly treatments of the grotesque, there is a certain amount of agreement as regard its essential nature. The grotesque emerges as a tense combination of attractive an repulsive elements, of comic and tragic aspects, of ludicrous and horrifying features. Emphasis can be placed on either the bright or the dark side (or pole) of the grotesque. "

22 Dominique Ielh (1993) parle d'ambivalence sur le mode de l'attirance et de la répulsion.

23 Selon Meindl (1996: 18), "the grotesque central characteristic, or effect, is self-contradiction, given the fact that it incorporates such opposites as laughter and anxiety - opposites that admit of marked shifts of emphasis towards one pole or the other." 
ainsi l'attention vers le fait que la conscience est, elle-même, une construction illusoire ("consciousness itself is an illusory construct").

Par le biais du jeu avec l'illusion fictionnelle, le fantastique et le grotesque partagent des éléments ou composants similaires et se contaminent dans une réciprocité facile et agile, cheminant de pair très souvent.

Les exemples sont multiples et parmi eux — et pour s'en tenir aux auteurs choisis ici — c'est impossible de ne pas penser à La Métamorphose, au Rapport à une académie (narré par un singe), à certaines narratives de l'Histoire Universelle de l'Infamie, à certains contes de L'Aleph, etc.

Mais un des plus poignants exemples de glissement du grotesque au fantastique et d'interpénétration de leurs aspects (cheminant vers une fluide et étrange fusion des deux catégories ou prédicats) on peut le retrouver dans le roman de Miguel Ángel Asturias intitulé Monsieur Le Président. Lécrivain guatemaltèque réussi à créer une atmosphère irréelle et lyrique à partir d'une panoplie de personnages et situations lourdement grotesques. En effet, si le roman fait le tableau de la pauvreté et de la souffrance d'un peuple subjugué à la volonté d'un dictateur cruel et instable, le tableau présente une étrange intensité, parce que l'auteur en enfonce vraiment les couleurs de sa peinture en mettant l'accent précisément sur la représentation des plaies, de la laideur des pauvres, de la maladie, de la cruauté et de la torture, mais d'une façon stylisée et dépurée. En fait, l'auteur mène le laid et le grotesque si loin qu'il devient tragique et, par une utilisation innovatrice ${ }^{24}$ d'un discours plein de métaphores inusuelles et modernes, il atteint une atmosphère lyrique et fantasmagorique inoubliable et inégalable. Le recours à la représentation de rêves, de cauchemars et d'états de semi-conscience (causés par la fièvre, la maladie, la faim ou la torture) se mêle à la représentation de croyances mythiques indigènes ou catholiques et de ce fait il touche le "real maravilhoso". Mais au-delà de ces stratégies-ci, l'auteur travaille beaucoup ce qu'il désigne par la "disproportion fantastique" advenue de la "dissociation des idées" et de "l'élasticité du monde dans les miroirs"25, ce qui

24 L'auteur atteint un rythme discoursif au même temps rapide et flou plein de onamatopées: “... Erre, erre,ere.../ I-N-R-Idiota! / I-N-R-Idiota! /... Erre, erre,ere.../ El afilador se afila los dientes oara reíse! Afiladores de risa! Dientes del afilador! "(Asturias, 1985: 240).

25 Il s'agit d'une description de la souffrance du protagoniste: "Las unãs aceradas de la fiebre le aserraban la frente. Disociación de ideas. Elasticidad del mundo en los espejos. Desproporción fantástica. Huracán delirante. Fuga vertiginosa, horizontal, vertical, oblicua, recién nacida y muerta en espiral...

Curvadecurvaencurvadecurvacurvade curvaencurvala mujer de Lot." (Asturias, 1985: 40) 
lui permet de construire des phrases au même temps lyriques et irréelles: "Atardició. Cielo verde. Campo verde." (Asturias, 1985: 239). Cette phrase synesthésique montre cette «élasticité» miroitante qui caractérise les descriptions de l'espace où se meuvent les personnages du roman (chapitre III).

L'intensité de l'ouverture du roman est impressionnante: la persécution et la fuite du personnage Épouvantail ${ }^{26}$ — un mendiant retardé, idiot, hanté par la nostalgie de sa mère. Le lecteur accompagne le chaos de ses émotions — c'est à dire, le lecteur est presque entraîné au-dedans de sa folie et de ses visions. Dans une de ses visions (au chapitre IV), par exemple, il écoute un oiseau qui est aussi une cloche qui dit:

— Je suis la Pomme-Rose de l'Oiseau du Paradis, je suis la vie, moitié de mon corps est mensonge l'autre moitié vérité; je suis la rose et la pomme, à tous je donne un œil de verre e un œil de vérité; ceux qui voient avec mon œil de verre voient parce qu'ils rêvent, ceux qui voient avec mon oeil de vérité voient parce qu'ils regardent! Je suis la vie, la Pomme-Rose de l'Oiseau du Paradis; je suis le mensonge de toutes les choses réelles, la réalité de toutes les fictions! (Asturias, 1985: 27). ${ }^{27}$

On peut noter ici une ambivalence multiple: un animal parlant qui alerte comme une cloche; un oiseau fantastique mixte de pomme et rose, abstraitement divisé en mensonge et vérité, qui grotesquement nous offre un oil de verre. Par le biais de la double appartenance catégorielle l'objet est perçu comme fantastique; par le biais de l'abstraction le discours devient lyrique et allégorique.

L’indécidabilité et l'ambivalence viennent aussi du personnage principal, Cara de Ángel, tantôt ange tantôt démon pendant une longue part du roman, montrant ainsi allégoriquement l'argile de laquelle l'homme est fait. L'incertitude du fantastique rend le grotesque et le tragique de ce roman moins lourd et toutes

26 Il s'agit d'une traduction approximative une fois que la nom en espagnol est Pelele et il se rapporte à un petit bonhomme de paille pour jouer pendant le Carnaval.

27 Original en espanhol: “- Soy la Manzana-Rosa del Ave del Paraíso, soy la vida, la mitad de mi cuerpo es mentira y la mitad es verdad; soy rosa y son manzana, doy a todos un ojo de vidrio y un ojo de verd: los que ven con mi ojo de vidrio ven porque sueñam, los que ven com mi ojo de verdad ven porque miran! Soy la vida, la Manzana Rosa del Ave del Paraíso; soy la mentira de todas las cosas reales, la realidad de todas las ficciones!" (Asturias, 1985: 243). 
catégories esthétiques participent au sens allégorique du roman lui donnant une profondeur et une complexité hors du commun.

Dans les auteurs cités il est commun cette contamination du fantastique et du grotesque, mais il y a certainement d'autres catégories ou prédicats esthétiques qui contribuent au fantastique, ce qui laisse ouvert le processus de compréhension du fantastique et permet de refaire interminablement la question s'il y aura jamais une seule clé pour le fantastique.

\section{BIBLIOGRAPHIE}

ANDERSON, James. (1985). "Aesthetic Concepts of Art". Theories Of Art Today. Ed. Noël CARROLL. Madison/Wisconsin: The University of Wisconsin Press, 2000. 65-87.

ASTURIAS, Miguel Ángel. (1968). El Senõr Presidente. En Tres Obras. 3ème ed. Caracas: Fundación Biblioteca Ayacuchio.

BLANCHÉ, Robert. (1979). Catégories esthétiques. Paris: Librairie Philosophique J. Vrin.

BORGES, Jorge Luis. (1998). "A arte narrativa e a magia" (1932). Obras Completas. Vol. I. Lisboa: Editorial Teorema.

(1998) "Tlön, Uqbar, Orbis Tertius" (1932). Obras Completas. Vol. I. Lisboa: Editorial Teorema.

, (1985) "Kafka y sus precursores" (1959). Outras Inquisiones. Prosa Completa. Vol. 3. Barcelona: Brugera.

BOZZETTO, Roger. (2002). "Fantastique et real maravilloso: le domaine latino-americain". Territoires des fantastiques. Aix-en-Provence: Presses de l'Université de Provence, 94-116. Partiellement disponible en Réflexions sur le Fantastique. 2004, http:// www.noosfere.com/Bozzetto/

BOZZETTO, Roger et HUFTIER, Arnaud. (2004). Les Frontières du Fantastique. Approches de l'impensable en littérature. Valenciennes: Presses Universitaires de Valenciennes.

CHANDLER, Daniel. "The Active Viewer. Context ans Expectations; Categorization and Selectivity». http://www.aber.ac.uk/media/Modules/MAinTV/visper05.html. Publié en juillet 1997, modifié en décembre 2004, consulté en septembre 2008.

CHAREYRE-MEJAN, Alain. (1998). Le Réel et le Fantastique. Paris: L'Harmattan.

DUBOST, Francis. (1991). Aspects Fantastiques de la Littérature Narrative Médievale. Paris: Librarie Honoré Champion Éditeur.

GENETTE, Gérard. (1994). L'Euvre de l'Art. Immanence et Transcendance. Vol. I. Paris: Ed. Seuil.

, (1997). L'Cuvre de l'Art. La Relation Esthétique. Vol. II. Paris: Seuil.

GOLDman, Alan H. (1995). Aesthetic Value. Boulder, Colorado: Westview Press.

KAFKA, Franz. (1945) La Colonie Pénitentiaire. Nouvelles suivies d'un Journal Intime. Paris: Egloff. 
KANT, Immanuel. (2004), Critique of Aesthetic Judgement. Trad. de James Creed Meredith. Electronic Text Center, University of Virginia Library. Consulté en 17-8-2008. <http:// etext.lib.virginia.edu/toc/modeng/public/KanJudg.html>.

HUFTIER, Arnaud. (2007). "Fantastique, fantastic, fantastiche, fantástica, fantastico. Derivas ocidentais de uma palavra”. O Fantástico. Coord. Maria João Simões, Coimbra: CLP. 23-41.

IEHL, Dominique. (1993). "Grotesque, ambivalence, indétermination". Les Cahiers du Cerli 3, Automne: 11-18.

MEINDL, Dieter. (1996). American Fiction and The Metaphysics of the Grotesque. Columbia: University Missouri Press.

MOLINO, Jean. "Trois modèles d'analyse du fantastique". Europe. Les Fantastiques. $58^{\circ}$ Année, no 611, Mars (1980): 12-26. Disponible partiellement en Index Critique de la Littérature Fantastique: <http://indexfantastique.phpnet.org/Textes/FicheTxt.ph p3?key=1054\&PHPSESSID=4c50d487ddf48010f6ceda6e865c3998 >.

MORIN, Edgar. (1991). "De la complexité: complexus". Les Théories de la Complexité. Autour de l'Euvre d'Henri Atlan (Colloque de Cerisy). Ed. Françoise Soulié. Paris: Seuil, 283-296.

SIBER, Sharon L. (2003). "Time and the Fantastic: Simultaneity in Borges, Cortázar, Lezama Lima, and Paz". Fantastic Odysseys. Selected Essays From the Twenty-Second International Conference of the Fantastic in the Arts. Ed. Mary PHARR. West Port: Praeger, 23-28.

SIMÕES, Maria João. (2006). "Diafaneidade e fronteiras: o fantástico em António Vieira, M. G. Llansol e Mário de Carvalho”. Olhares sobre o Fantástico. Coord. Henriqueta Gonçalves,Vila Real: Pena Perfeita, 17-32.

, (2007) "Fantástico como categoria estética: diferenças entre os monstros de Ana Teresa Pereira e Lídia Jorge”. O Fantástico. Coord. Maria João SIMÕES. Coimbra: CLP, 65-81.

SOURIAU, Étienne. (1990). Vocabulaire d'Esthétique. Paris: PUF.

WALTON, Kendall. "Appreciating Fiction: Suspending Disbelief or Pretending Belief". Dispositio. 5, $\mathrm{n}^{\circ}$ 13-14 (1980): 1-18. (Trad. fr.: Walton, Kendall. "Comment on apprécie la fiction". Agone. 14 (1995): 15-47.

, (1990), Mimesis as Make-Believe. On the Foundations of the Representational Arts. Cambridge Mass.: Havard University Press.

ZIEGLER, James. (1985). "Primitive, Newtonian, and Einsteinian Fantasies: three Worldviews". The Scope of the Fantastic. Theory, Technique, Major Authors. Eds., Robert COLLINS \& Howard PEARCE. West Port/Conneticut/London: Greenwood Press. 